\title{
Outage Performance for Dynamic Power Allocation in Hybrid Non-orthogonal Multiple Access Systems
}

\author{
Zheng Yang, Zhiguo Ding, Senior Member, IEEE, and Pingzhi Fan, Fellow, IEEE, Zheng Ma, Member, IEEE
}

\begin{abstract}
In this letter, we propose a novel dynamic power allocation scheme for hybrid downlink non-orthogonal multiple access (DH-NOMA). The exact expressions for the outage probabilities and their asymptotic approximations at high signal-tonoise ratio are derived in DH-NOMA systems. Both Monte Carlo simulations and analytical results are used to compare the proposed scheme with existing works, such as NOMA based on fixed power allocation (F-NOMA) and cognitive radio inspired NOMA (CR-NOMA). The provided results demonstrate that the DHNOMA scheme can always achieve better outage performance than traditional orthogonal multiple access, and achieve a more balanced trade-off between the two user individual rates than F-NOMA and CR-NOMA.
\end{abstract}

Index Terms-Non-orthogonal multiple access, successive interference cancellation, outage probability.

\section{INTRODUCTION}

Non-orthogonal multiple access (NOMA) can efficiently improve the average throughput and the cell-edge throughput in cellular networks, which is reason why it has been considered as a candidate in the fifth generation networks [1]. The key idea of NOMA is that the power domain is used to realize multiple access and serve multiple users with different channel conditions, where the users with strong channel conditions obtain less power than the users with weak channel conditions, and successive interference cancellation (SIC) is applied at the receiver side [2].

In [3], the authors have investigated the performance for downlink coordinated NOMA systems, while uplink NOMA has been studied in [4]. The outage performance for users with random locations in NOMA systems based on fixed power allocation (F-NOMA) has been considered in [2], which demonstrates that NOMA can achieve better performance than orthogonal multiple access (OMA). However, when the users' target rate and the power allocation factor are not correctly chosen, outage events always occur. In [5], the authors have studied the outage performance for the paired users in cognitive radio inspired NOMA (CR-NOMA), where the user with worse channel conditions is regarded as a primary user. However, the disadvantage of CR-NOMA is that the strong user's diversity gain entirely depends on the weak user's channel quality.

In this letter, a new dynamic power scheme based on hybrid NOMA (DH-NOMA) is proposed. The key feature of the DHNOMA is that when the strong user's channel gain is worse

Z. Yang, P. Fan, and Z. Ma are with the Institute of Mobile Communications, Southwest Jiaotong University, Chengdu 610031, P. R. China. (email: zyfjnu@163.com,p.fan@ieee.org, zma@home.swjtu.edu.cn).

Z. Ding is with the School of Computing and Communications, Lancaster University, LA1 4YW, UK. (e-mail: z.ding@lancaster.ac.uk).

This work was supported by National Natural Science Foundation of China (No. 61471302), National Sci \& Tech Major Project (No. 2016ZX03001018002), National High-tech R \& D Program of China (No. 2014AA01A707), and 111 project (No. 111-2-14). than a threshold determined by the weak user's fixed target rate, traditional OMA is applied to serve users; otherwise, NOMA is applied. Based on the proposed DH-NOMA scheme, the exact expressions for the outage probabilities and their asymptotic approximations for the paired users are derived. Compared with OMA, DH-NOMA always outperforms OMA for different system parameters. Recall that the users' outage probabilities in F-NOMA tend to one if the power allocation factor and the targeted data rate are chosen in a wrong way [2], while the strong user's diversity gain suffers loss in CR-NOMA [5]. The DH-NOMA can perfectly avoid these disadvantages, and achieve a more balanced trade-off between the two user individual rates compared to F-NOMA and CRNOMA.

\section{SySTEM MOdEL}

Consider a downlink NOMA network with $M$ single antenna users and one single antenna base station. Without loss of generality, we order the users according to their channel conditions as follows: $\left|h_{1}\right|^{2} \leq \cdots \leq\left|h_{M}\right|^{2}$, where $h_{i}$ follows from Rayleigh fading. In this letter, the $p$-th user and the $q$-th user are paired to perform NOMA, which is motivated by the fact that the two-user form of NOMA has been included in in Third Generation Partnership Project (3GPP) - Long Term Evolution (LTE) Advanced recently [6].

According to the NOMA scheme, decode at each receiver is conducted always from the weaker user to the better user. Therefore, the rate of the $p$-th user is given by [2]

$$
R_{p}^{N}=\log _{2}\left(1+\frac{\alpha_{p}\left|h_{p}\right|^{2}}{\alpha_{q}\left|h_{p}\right|^{2}+1 / \rho}\right),
$$

where $\rho$ is the transmit signal-to-noise ration (SNR), $\alpha_{p}$ and $\alpha_{q}$ are the power allocation factors for the $p$-th user and $q$-th user, respectively, and $\alpha_{p}>\alpha_{q}, \alpha_{p}+\alpha_{q}=1$.

Note that SIC is applied at the $q$-th user so that it can decode the $p$-th user's message, before detecting its own message. The rate to detect the $p$-th user's message at the $q$-th user can be calculated as $R_{q \rightarrow p}^{N}=\log _{2}\left(1+\frac{\alpha_{p}\left|h_{q}\right|^{2}}{\alpha_{q}\left|h_{q}\right|^{2}+1 / \rho}\right)$. Since the fact that $\left|h_{p}\right|^{2} \leq\left|h_{q}\right|^{2}$, we can ensure that $R_{q \rightarrow p}^{N} \geq R_{p}^{N}$, i.e., SIC is always correctly applied at the $q$-th user. Therefore, the $q$-th user can remove the message from the $p$-th user, and the rate of the $q$-th user is given by

$$
R_{q}^{N}=\log _{2}\left(1+\alpha_{q} \rho\left|h_{q}\right|^{2}\right) .
$$

Furthermore, if the $p$-th user's target rate, $R_{p}$, is determined by a predefined quality of service (QoS) requirement, i.e., $R_{p}$ is a preset rate. In this case, we cannot ensure that $\operatorname{Pr}\left\{R_{q \rightarrow p}^{N} \geq\right.$ $\left.R_{p}\right\}=1$, i.e., SIC cannot always be correctly carried out at the $q$-th user. In order to ensure SIC can be correctly carried out at the $q$-th user, we constraint

$$
\log _{2}\left(1+\frac{\alpha_{p}\left|h_{q}\right|^{2}}{\alpha_{q}\left|h_{q}\right|^{2}+1 / \rho}\right) \geq R_{p}
$$

This means the constraint condition of $\alpha_{q}$ is given by

$$
\alpha_{q} \leq \frac{Y^{2}-\varepsilon_{p}}{\varepsilon_{p}\left(Y^{2}-1\right)}, Y>\sqrt{\varepsilon_{p}},
$$


where $Y=\sqrt{1+\rho\left|h_{q}\right|^{2}}$, and $\varepsilon_{p}=2^{R_{p}}$.

Consider that the $q$-th user's rate has a dynamic QoS requirement as follows:

$$
\log _{2}\left(1+\alpha_{q} \rho\left|h_{q}\right|^{2}\right) \geq \frac{1}{2} \log _{2}\left(1+\rho\left|h_{q}\right|^{2}\right),
$$

which means that the $q$-th user is allowed to perform NOMA only when it can achieve a better rate than OMA. Then, the constraint condition of $\alpha_{q}$ can be derived as

$$
\alpha_{q} \geq \frac{1}{Y+1} .
$$

Similarly to the $q$-th user, we also assume that the $p$-th user's rate is to meet a dynamic QoS constraint as follows:

$$
\log _{2}\left(1+\frac{\alpha_{p}\left|h_{p}\right|^{2}}{\alpha_{q}\left|h_{p}\right|^{2}+1 / \rho}\right) \geq \frac{1}{2} \log _{2}\left(1+\rho\left|h_{p}\right|^{2}\right) .
$$

This means the constraint condition of $\alpha_{q}$ can be written as

$$
\alpha_{q} \leq \frac{1}{X+1},
$$

where $X=\sqrt{1+\rho\left|h_{p}\right|^{2}}$.

From (4) and (6), it should be guaranteed that $\frac{Y^{2}-\varepsilon_{p}}{\varepsilon_{p}\left(Y^{2}-1\right)} \geq$ $\frac{1}{Y+1}$, which implies

$$
Y \geq \varepsilon_{p} .
$$

It can be observed from (9) that the paired users are allowed to perform NOMA under the condition of $Y>\varepsilon_{p}$. Or in other words, when $X \leq Y \leq \varepsilon_{p}$, NOMA cannot outperform OMA. In this case, we adopt OMA, such as time division multiple access (TDMA), to serve the paired users. Therefore, a hybrid NOMA is proposed to serve paired users in this letter.

Based on (4), (6) (8), and (9), we can use the parameter $\beta, 0 \leq \beta \leq 1$ to tune the range of $\alpha_{q}$ as given in the following:

$$
\alpha_{q}=\frac{\beta}{Y+1}+\min \left\{\frac{1-\beta}{X+1}, \frac{(1-\beta)\left(Y^{2}-\varepsilon_{p}\right)}{\varepsilon_{p}\left(Y^{2}-1\right)}\right\},
$$

where $Y>\varepsilon_{p}$, and it is noted that $\alpha_{q}<\frac{1}{2}$.

It can be seen from (10) that $\alpha_{q}$ is a decreasing function of $\beta$. In other words, $\beta$ is a dynamic factor, which can determine the range of $\alpha_{q}$. Furthermore, both the rates of user $p$ and $q$ are functions of $\alpha_{q}$, which means different choices of $\beta$ will affect the performance at user $p$ and $q$.

From (1) and (10), we can see that $R_{p}^{N}$ is an increasing function of $\beta$, and therefore the minimal rate for user $p$ with $\beta=0$ is given by

$$
R_{p}^{N, \text { min }}=\log _{2} X^{2}-\log _{2}\left(1+\alpha_{q}^{0}\right) \geq \frac{1}{2} \log _{2}\left(1+\rho\left|h_{p}\right|^{2}\right),
$$

where $\alpha_{q}^{0}=\min \left\{\frac{X^{2}-1}{X+1}, \frac{\left(X^{2}-1\right)\left(Y^{2}-\varepsilon_{p}\right)}{\varepsilon_{p}\left(Y^{2}-1\right)}\right\}$.

From (2) and (10), we can see that $R_{q}^{N}$ is a decreasing function of $\beta$, and then the minimal rate for user $q$ with $\beta=1$ is given by

$$
R_{q}^{N, \min }=\frac{1}{2} \log _{2}\left(1+\rho\left|h_{q}\right|^{2}\right) .
$$

One can observe from (11) and (12) that user $p$ and user $q$ in the proposed DH-NOMA scheme can always achieve better performance than those in OMA. Furthermore, different fairness can be realized between the two users by adjusting $\beta$.

\section{OUtage Probability FOR DH-NOMA}

In this section, we focus on the outage probability for the paired users in DH-NOMA systems.

An exact expressions for the outage probability of user $q$ and its diversity gain are given in the following theorem.
Theorem 1: The outage probability of the $q$-th user in DH-NOMA systems is given by

$$
\begin{aligned}
\mathrm{P}_{q} & =\varpi \sum_{i=0}^{p-1} \sum_{j=0}^{q-p-1} \frac{(-1)^{i+q-p-1-j}\left(\begin{array}{c}
p-1 \\
i
\end{array}\right)\left(\begin{array}{c}
q-p-1 \\
j
\end{array}\right)}{i+j+1} \\
& \times\left(\int_{0}^{\epsilon_{2}} e^{-(M-p-j) y}\left(1-e^{-(i+j+1) g_{2}(y)}\right) d y\right. \\
& \left.+\int_{0}^{\epsilon_{7}} e^{-(M-p-j) y}\left(e^{-(i+j+1) g_{2}(y)}-e^{-(i+j+1) y}\right) d y\right),
\end{aligned}
$$

where $\quad \varpi=\frac{M !}{(p-1) !(q-p-1) !(M-q) !}, \quad \epsilon_{2}=\frac{\kappa_{1}^{2}-1}{\rho}, \quad \kappa_{1}=$ $\frac{-\beta \varepsilon_{p}+\sqrt{\beta^{2} \varepsilon_{p}^{2}+4(1-\beta) \varepsilon_{p} \varepsilon_{q}}}{2(1-\beta)}, \epsilon_{7}=\frac{\varepsilon_{q}^{2}-1}{\rho}, g_{2}(y)=\frac{G_{2}^{2}(Y)-1}{\rho}, G_{2}(Y)=$ $\frac{(1-\beta)\left(Y^{2}-1\right)}{\varepsilon_{q}-1-\beta(Y-1)}-1$. And, the diversity gain achieved by the $q$-th user in DH-NOMA systems is $q$.

Proof: See Appendix.

With the similar steps to those in theorem 1 , we can obtain the outage performance for the $p$-th user in DH-NOMA systems as follows:

$$
\begin{aligned}
\mathrm{P}_{p} & =1-\varpi \sum_{i=0}^{p-1} \sum_{j=0}^{q-p-1} \frac{(-1)^{i+q-p-1-j\left(\begin{array}{c}
p-1 \\
i
\end{array}\right)\left(\begin{array}{c}
q-p-1 \\
j
\end{array}\right)}}{i+j+1} \\
& \times\left(\int_{\epsilon_{6}}^{\infty} e^{-(M-p-j) y-(i+j+1) g_{4}(y)} d y-\frac{e^{-(M-p+i+1) \epsilon_{6}}}{M-p+i+1}\right),
\end{aligned}
$$

where $g_{4}(y)=\frac{G_{4}^{2}(Y)-1}{\rho}, G_{4}(Y)=\frac{\varepsilon_{p}(1-\beta)(Y+1)+\sqrt{\Delta}}{2\left(Y-\beta \varepsilon_{p}+1\right)}, \Delta=$ $\left(\varepsilon_{p}(1-\beta)(Y+1)\right)^{2}+4 \beta \varepsilon_{p} Y\left(Y-\beta \varepsilon_{p}+1\right), \epsilon_{6}=\frac{\varepsilon_{p}^{2}-1}{\rho}$. Furthermore, the diversity order of the $p$-th user is $p$.

Remark 1: The results in (13) and (14) demonstrate that the users in DH-NOMA can achieve the same diversity gain as in F-NOMA [2], while avoiding the problem that the outage probability always becomes one. Furthermore, DH-NOMA can ensure the $q$-th user achieves the full diversity gain, while the $q$-th user only achieves a diversity gain of $p$ in CR-NOMA [5].

\section{NumericAl Results}

Monte Carlo simulations are provided to verify the accuracy of the developed analytical results in this section. The parameters used in the simulations are set as follows. All the channels are assumed to be independent and identically distributed complex Gaussian distributed with mean zero and variance one.

Fig. 1 shows that the outage probabilities of the paired users in different NOMA systems as a function of SNR. One can observe that for F-NOMA, the outage probabilities for the $q$-th user and the $p$-th user in Fig. 1(a) and Fig. 1(b), respectively, are always one when the users' target rates $R_{p}=2$ bits $/ \mathrm{s} / \mathrm{Hz}, R_{q}=3.5$ bits $/ \mathrm{s} / \mathrm{Hz}$. This is because the users' target rates and power allocation factors are not correctly chosen [2]. Therefore, F-NOMA cannot always ensure its outage performance is superior to OMA.

For CR-NOMA, the $p$-th user can always achieve better outage performance than that in OMA in Fig. 1(b). However, CR-NOMA leads to a loss of the diversity gain for the $q$ th user's diversity gain loss compared to that in OMA in Fig. 1(a). The main reason is that the $p$-th user is considered as the primary user, while the $q$-th user is regarded as the secondary user in CR-NOMA [5]. Therefore, for CR-NOMA, the $q$-th user's outage probability is worse than than that in OMA, in 


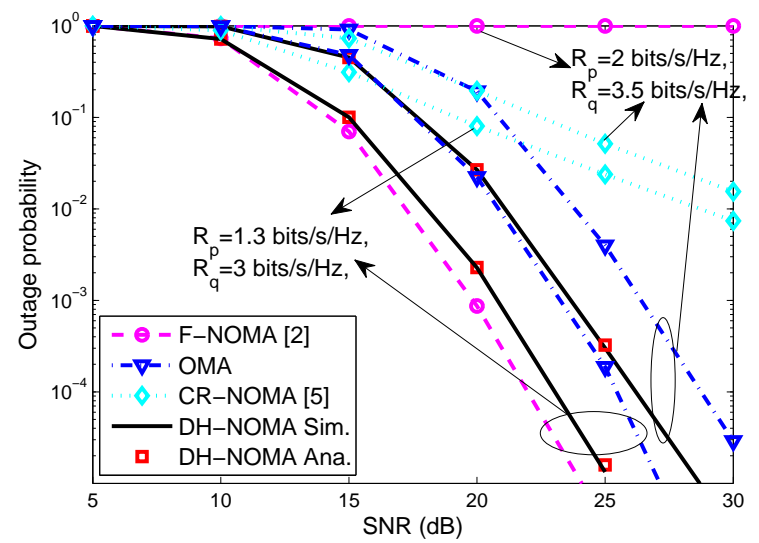

(a) The $q$-th user.

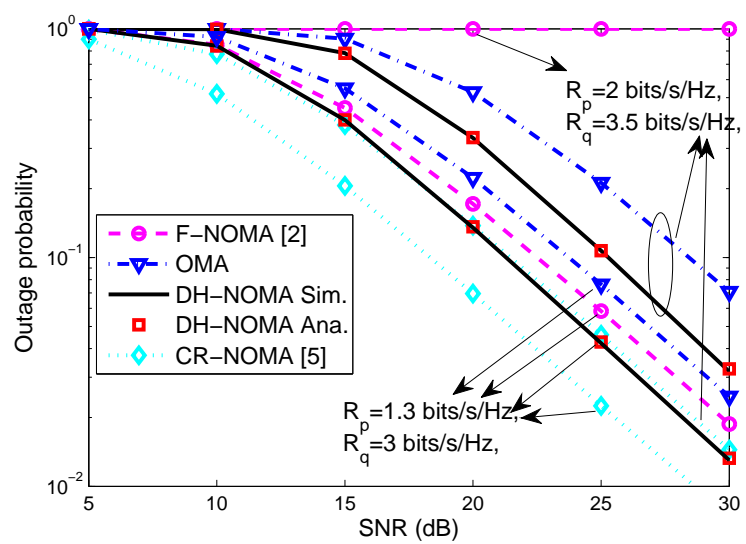

(b) The $p$-th user

Fig. 1. Compare with the outage probability for users in different NOMA systems, where $\beta=0.5, M=5, p=1, q=5$, and $\alpha_{q}=0.25$ for F-NOMA.

order to ensure that the $p$-th user's can achieve better outage performance compared to OMA.

For DH-NOMA, although the outage performance for the $p$-th user is worse than those in CR-NOMA, it can be seen that the outage probabilities for the $p$-th user and the $q$-th user are always superior to those in OMA with different system parameters in Fig. 1(a) and Fig. 1(b). This means that DH-NOMA can achieve better fairness than F-NOMA and CR-NOMA. In addition, it is worth pointing out that the analytical results in (13) and (14) are matched quite well with the computer simulations.

In Fig. 2, the average rates of the $p$-th user and the $q$-th user in DH-NOMA and other multiple access schemes are shown as a function of SNR. It can be seen from Fig. 2, both the paired users in DH-NOMA can achieve higher rates than those in OMA. Furthermore, the average rate of the $q$-th user in both CR-NOMA and F-NOMA are larger than that in OMA, while the $p$-th user in both CR-NOMA and F-NOMA achieve a less average rate than that in OMA. Therefore, DH-NOMA can achieve a better tradeoff between data rates and fairness than the other multiple access schemes.

\section{CONCLUSiOnS}

In this letter, a novel DH-NOMA has been introduced. We have studied the outage performance for the paired users in

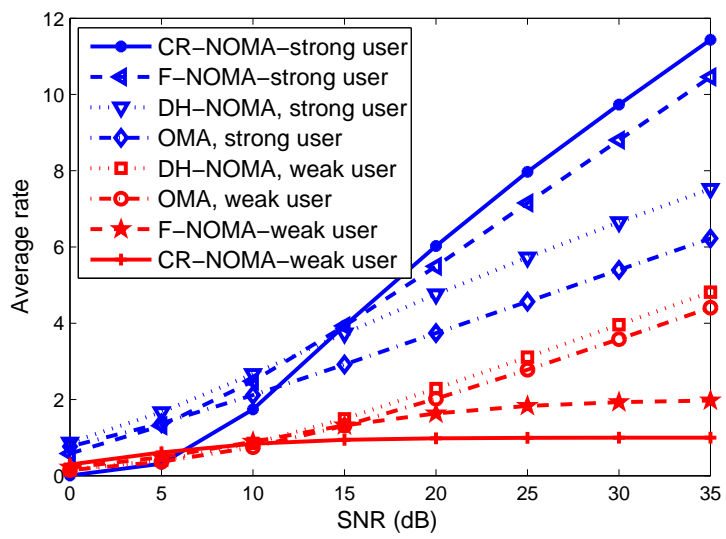

Fig. 2. The average rate for strong user and weak user in DH-NOMA systems compare to other multiple access schemes, where $M=4$, $p=1, q=4$, and $\beta=0.6$. For F-NOMA, $\alpha_{w}=0.25$; For CRNOMA, $R_{v}=1$ bits $/ \mathrm{s} / \mathrm{Hz}$.

DH-NOMA systems. The developed and numerical results showed that DH-NOMA is always superior to the OMA, and achieves a more balanced trade-off between the two user individual rates than F-NOMA and CR-NOMA. In this letter, we have considered to select two users to perform DH-NOMA, and one promising future work is to extend the DH-NOMA to more than two users, where one user can be regarded as a primary user, and the others are considered as secondary users.

\section{APPENDIX}

i) When $Y>\varepsilon_{p}$, the paired users are selected for the implementation of NOMA. Based on the relationship between $\frac{1}{X+1}$ and $\frac{Y^{2}-\varepsilon_{p}}{\varepsilon_{p}\left(Y^{2}-1\right)}$, the power allocation factor $\alpha_{q}$ in (10) can be classified as follows:

1) If $\frac{1}{X+1}>\frac{Y^{2}-\varepsilon_{p}}{\varepsilon_{p}\left(Y^{2}-1\right)}$, the power allocation factor $\alpha_{q}$ in (10) can be rewritten as

$$
\alpha_{q}=\frac{\beta}{Y+1}+\frac{(1-\beta)\left(Y^{2}-\varepsilon_{p}\right)}{\varepsilon_{p}\left(Y^{2}-1\right)},
$$

which needs to meet the following two constraints: a) $X<$ $\varepsilon_{p}-1$, and $Y>\varepsilon_{p}$; b) $\varepsilon_{p}-1<X<\varepsilon_{p}$, and $\varepsilon_{p}<Y<\sqrt{\frac{\varepsilon_{p} X}{X+1-\varepsilon_{p}}}$.

2) If $\frac{1}{X+1} \leq \frac{Y^{2}-\varepsilon_{p}}{\varepsilon_{p}\left(Y^{2}-1\right)}$, the power allocation factor $\alpha_{q}$ in (10) can be rewritten as

$$
\alpha_{q}=\frac{\beta}{Y+1}+\frac{1-\beta}{X+1},
$$

which needs to meet the following two constraints: c) $Y>\varepsilon_{p}$, $\frac{\left(\varepsilon_{p}-1\right) Y^{2}}{Y^{2}-\varepsilon_{p}}<X<\varepsilon_{p}$; d) $Y \geq X \geq \varepsilon_{p}$.

Based on (15), the coverage probability of the $q$-th user in NOMA systems is given by

$$
\begin{aligned}
\overline{\mathrm{P}}_{q, 1}^{N} & =\operatorname{Pr}\left\{\log _{2}\left(1+\alpha_{q} \rho\left|h_{q}\right|^{2}\right)>R_{q}\right\} \\
& =\operatorname{Pr}\left\{(1-\beta) Y^{2}+\beta \varepsilon_{p} Y-\varepsilon_{p} \varepsilon_{q}>0\right\}=\operatorname{Pr}\left\{Y>\kappa_{1}\right\} .
\end{aligned}
$$

Recall that the above $\overline{\mathrm{P}}_{q, 1}^{N}$ has to meet both conditions: a) $X<\varepsilon_{p}-1$, and $Y>\varepsilon_{p}$; b) $\varepsilon_{p}-1<X<\varepsilon_{p}$, and $\varepsilon_{p}<Y<$ $\sqrt{\frac{\varepsilon_{p} X}{X+1-\varepsilon_{p}}}$. Therefore, the $\overline{\mathrm{P}}_{q, 1}^{N}$ can be rewritten as

$$
\begin{aligned}
\overline{\mathrm{P}}_{q, 1}^{N} & =\underbrace{\operatorname{Pr}\left\{X<\varepsilon_{p}-1, Y>\kappa_{1}\right\}}_{Q_{1}} \\
& +\underbrace{\operatorname{Pr}\left\{\varepsilon_{p}-1<X<G_{3}(Y), Y>\kappa_{1}\right\}}_{Q_{2}} .
\end{aligned}
$$


Note that the joint probability density function (PDF) of $\left|h_{p}\right|^{2}$ and $\left|h_{q}\right|^{2}$ is given by [7]

$$
\begin{array}{r}
f_{\left|h_{p}\right|^{2},\left|h_{q}\right|^{2}}(x, y)=\varpi(F(x))^{p-1}(F(y)-F(x))^{q-p-1} \\
\times(1-F(y))^{M-q} f(x) f(y), 0<x<y,
\end{array}
$$

where $f(x)=e^{-x}$ and $F(x)=1-e^{-x}$.

Based on (19), $Q_{1}$ in (18) can be evaluated as

$$
\begin{aligned}
Q_{1}= & \varpi \sum_{i=0}^{p-1} \sum_{j=0}^{q-p-1}(-1)^{i+q-p-1-j}\left(\begin{array}{c}
x-1 \\
i
\end{array}\right)\left(\begin{array}{c}
q-p-1 \\
j
\end{array}\right) \\
& \times \int_{0}^{\epsilon_{1}} e^{-(i+j+1) x} d x \int_{\epsilon_{2}}^{\infty} e^{-(M-p-j) y} d y \\
= & \varpi \sum_{i=0}^{p-1} \sum_{j=0}^{q-p-1} \frac{(-1)^{i+q-p-1-j}}{(i+j+1)(M-p-j)}\left(\begin{array}{c}
p-1 \\
i
\end{array}\right)\left(\begin{array}{c}
q-p-1 \\
j
\end{array}\right) \\
& \times\left(1-e^{-(i+j+1) \epsilon_{1}}\right) e^{-(M-v-j) \epsilon_{2}} .
\end{aligned}
$$

where $\epsilon_{1}=\frac{\left(\varepsilon_{p}-1\right)^{2}-1}{\rho}$.

Similarly to $Q_{1}^{\rho}, Q_{2}$ in (18) can be evaluated as

$$
\begin{aligned}
Q_{2}= & \varpi \sum_{i=0}^{p-1} \sum_{j=0}^{q-p-1} \frac{(-1)^{i+q-p-1-j}}{i+j+1}\left(\begin{array}{c}
p-1 \\
i
\end{array}\right)\left(\begin{array}{c}
q-p-1 \\
j
\end{array}\right) \\
& \times \int_{\epsilon_{2}}^{\infty}\left(e^{-(i+j+1) \epsilon_{1}}-e^{-(i+j+1) g_{3}(y)}\right) e^{-(M-p-j) y} d y .
\end{aligned}
$$

Based on (16), the coverage probability of the $q$-th user in NOMA systems is given by

$$
\begin{aligned}
\overline{\mathrm{P}}_{q, 2}^{N} & =\operatorname{Pr}\left\{\log _{2}\left(1+\alpha_{q} \rho\left|h_{q}\right|^{2}\right)>R_{q}\right\} \\
& =\operatorname{Pr}\left\{\beta(Y-1)+\frac{(1-\beta)\left(Y^{2}-1\right)}{X+1}>\varepsilon_{q}-1\right\} \\
& =\operatorname{Pr}\left\{Y>\kappa_{3}\right\}+\operatorname{Pr}\left\{\varepsilon_{p}<Y<\kappa_{3}, X<G_{2}(Y)\right\},
\end{aligned}
$$

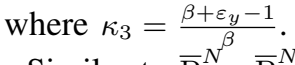

Similar to $\overline{\mathrm{P}}_{q, 1}^{N}, \overline{\mathrm{P}}_{q, 2}^{N}$ in (22) can be evaluated as

$$
\begin{aligned}
\overline{\mathrm{P}}_{q, 2}^{N} & =\varpi \sum_{i=0}^{p-1} \sum_{j=0}^{q-p-1} \frac{(-1)^{i+q-p-1-j}}{i+j+1}\left(\begin{array}{c}
p-1 \\
i
\end{array}\right)\left(\begin{array}{c}
q-p-1 \\
j
\end{array}\right) \\
& \times\left(\int_{\epsilon_{2}}^{\infty} e^{-(i+j+1) g_{3}(y)-(M-v-j) y} d y-\int_{\epsilon_{2}}^{\epsilon_{7}} e^{-(i+j+1) g_{2}(y)}\right. \\
& \left.\times e^{-(M-v-j) y} d y-\int_{\epsilon_{7}}^{\infty} e^{-(i+j+1) y-(M-v-j) y} d y\right) .
\end{aligned}
$$

ii) When $X \leq Y \leq \varepsilon_{p}$, the paired users are selected for the implementation of OMA. The coverage probability of the $q$-th user is given as

$$
\begin{aligned}
\overline{\mathrm{P}}_{q}^{T} & =\operatorname{Pr}\left\{\frac{1}{2} \log _{2}\left(1+\rho\left|h_{q}\right|^{2}\right)>R_{q}, X \leq Y \leq \varepsilon_{p}\right\} \\
& =\operatorname{Pr}\left\{Y>\varepsilon_{q}, X \leq Y \leq \varepsilon_{p}\right\}=0 .
\end{aligned}
$$

Furthermore, note that

$$
\varpi \sum_{i=0}^{p-1} \sum_{j=0}^{q-p-1} \frac{(-1)^{i+q-p-1-j}\left(\begin{array}{c}
p-1 \\
i
\end{array}\right)\left(\begin{array}{c}
q-p-1 \\
j
\end{array}\right)}{(M-p+i+1)(M-p-j)}=1 .
$$

Combining (20), (21) (23), (24) and (25), and note that $\mathrm{P}_{q}=$ $1-\overline{\mathrm{P}}_{q, 1}^{N}-\overline{\mathrm{P}}_{q, 2}^{N}-\overline{\mathrm{P}}_{q}^{T}$, the first part of the theorem is completed.

Recall that $Y \stackrel{q}{=} \sqrt{1+\rho y}$, and therefore the integral in the first sum term in (13) can be rewritten as

$$
\begin{aligned}
& \int_{0}^{\epsilon_{2}} e^{-(M-p-j) y}\left(1-e^{-(i+j+1) g_{2}(y)}\right) d y \\
& =\frac{2}{\rho} \int_{0}^{\kappa_{1}} Y e^{-(M-p-j) \frac{Y^{2}-1}{\rho}}\left(1-e^{-(i+j+1) \frac{G_{2}^{2}(Y)-1}{\rho}}\right) d Y \triangleq I_{1} .
\end{aligned}
$$

The above integral $I_{1}$ can be approximated by using Gauss-
Chebyshev integration [8],

$$
\begin{aligned}
I_{1} \approx & \frac{\pi \kappa_{1}}{\rho K} \sum_{k=1}^{K} z_{k} \sqrt{1-Y_{k}^{2}} e^{-(M-p-j) \frac{z_{k}^{2}-1}{\rho}} \\
& \times\left(1-e^{-(i+j+1) \frac{G_{2}^{2}\left(z_{k}\right)-1}{\rho}}\right),
\end{aligned}
$$

where $z_{k}=\frac{\kappa_{1}}{2}\left(1+Y_{k}\right), Y_{k}=\cos \left(\frac{2 k-1}{2 K} \pi\right)$, and $K$ is the number of terms included in the summation.

Since $\rho \rightarrow \infty$, the series expansion of exponential functions and binomial expand can be applied into (27), and first term in (13) denoted by $\mathrm{P}_{q, 1}$ can be further expressed as

$$
\begin{aligned}
\mathrm{P}_{q, 1} & \approx \frac{\pi \kappa_{1} \varpi(-1)^{q-p-1}}{K} \sum_{k=1}^{K} z_{k} \sqrt{1-Y_{k}^{2}} \sum_{l_{2}=1}^{\infty} \frac{\left(1-G_{2}^{2}\left(z_{k}\right)\right)^{l_{2}}}{l_{2} ! \rho^{l_{2}}} \\
& \times \sum_{i=0}^{p-1}\left(\begin{array}{c}
p-1 \\
i
\end{array}\right)(-1)^{i} \sum_{n_{2}=0}^{l_{2}-1}\left(\begin{array}{c}
l_{2}-1 \\
n_{2}
\end{array}\right) j^{n_{2}} \sum_{n_{2}=0}^{l_{2}-1-n_{2}}\left(\begin{array}{c}
l_{2}-1-n_{2} \\
n_{3}
\end{array}\right) i^{n_{3}} \\
& \times \sum_{l_{1}=1}^{\infty} \frac{\left(1-z_{k}^{2}\right)^{l_{1}-1}}{\left(l_{1}-1\right) ! \rho^{l_{1}}} \sum_{j=0}^{q-p-1}(-1)^{j}\left(\begin{array}{c}
q-p-1 \\
j
\end{array}\right) \sum_{n_{1}=0}^{l_{1}-1}\left(\begin{array}{c}
l_{1}-1 \\
n_{1}
\end{array}\right) \\
& \times(-1)^{n_{1}} j^{n_{1}}(M-p)^{l_{1}-1-n_{1}} .
\end{aligned}
$$

Based on the sum of the binomial coefficients [9, Eq. $(0.153)]$

$$
\sum_{i=0}^{p-1}\left(\begin{array}{cl}
p-1 \\
i
\end{array}\right)(-1)^{i} i^{n_{3}}= \begin{cases}0, & 0 \leq n_{3} \leq p-2 \\
(-1)^{p-1}(p-1) !, & n_{3}=p-1\end{cases}
$$

The $\mathrm{P}_{q, 1}$ in (28) can be further approximated as

$$
\begin{array}{r}
\mathrm{P}_{q, 1} \approx \frac{\pi \kappa_{1} \varpi(-1)^{q-p}}{p K \rho^{q}} \sum_{k=1}^{K} z_{k} \sqrt{1-Y_{k}^{2}}\left(G_{2}^{2}\left(z_{k}\right)-1\right)^{p} \\
\times\left(z_{k}^{2}-1\right)^{q-p-1} .
\end{array}
$$

Similar, the second term in (13) denoted by $\mathrm{P}_{q, 2}$ can be also approximated as

$$
\mathrm{P}_{q, 2} \approx \frac{C}{\rho^{q}}
$$

where $C$ is a constant.

Combined (30) with (31), the second part of the theorem is completed.

\section{REFERENCES}

[1] "Proposed solutions for new radio access," Mobile and wireless communications enablers for the 2020 information society (METIS), Deliverable D.2.4, Feb. 2015.

[2] Z. Ding, Z. Yang, P. Fan, and H. V. Poor, "On the performance of nonorthogonal multiple access in 5G systems with randomly deployed users," IEEE Sig. Process. Lett., vol. 21, no. 12, pp. 1501-1505, Dec. 2014

[3] J. Choi, "Non-orthogonal multiple access in downlink coordinated twopoint systems," IEEE Commun. Lett., vol. 18, no. 2, pp. 313-316, Feb. 2014.

[4] M. Al-Imari, P. Xiao, M. A. Imran, and R. Tafazolli, "Uplink nonorthogonal multiple access for 5G wireless networks," in 11th International Symposium on Wireless Communications Systems (ISWCS), Aug. 2014, pp. 781-785.

[5] Z. Ding, P. Fan, and H. V. Poor, "Impact of user pairing on 5G nonorthogonal multiple access," IEEE Trans. Veh. Technol., to appear in 2016.

[6] 3rd Generation Partnership Project (3GPP), "Study on downlink multiuser superposition transmission for LTE," Shanghai, China, Mar. 2015.

[7] H. A. David and H. N. Nagaraja, Order Statistics. John Wiley, New York, 3rd ed., 2003.

[8] E. Hildebrand, Introduction to Numerical Analysis. Dover, New York, USA, 1987.

[9] I. S. Gradshteyn and I. M. Ryzhik, Table of Integrals, Series and Products, 6th ed. New York: Academic Press, 2000. 